\title{
Telomere measurement tools: Telometric produces biased estimates of telomere length
}

\section{Heredity (2011) 107, 371; doi:10.1038/hdy.2011.10; published online 23 March 2011}

Over the past decade, the fields of ecology and evolutionary biology have begun to explore the causes and consequences of telomere length variation, but, unfortunately, measuring telomeres is complicated. This has naturally resulted in a progression of new methodologies, as they are adopted, refined, and are sometimes discarded when better tools become available. Recent papers have alerted ecologists to potential methodological problems, and called for standardization in telomere measurement and analysis methodologies (Haussmann and Mauck, 2008; Monaghan, 2010). The recent review of current methodology by Horn et al. (2010) again emphasizes where caution is needed when measuring telomeres.

Here, we address the remarks made by Horn et al. (2010) regarding a telomere measurement tool-Telometric, an open-source Java program (Grant et al., 2001). Previously, we have stated that Telometric gives biased results compared to other telomere analysis methods as it uses an algorithm that generates interpolated data (Haussmann and Mauck, 2008; Salomons et al., 2009). Horn et al. (2010) state in response that 'their experience with Telometric and a review of the published literature in which Telometric has been used suggests that there is no support for this claim', but to the best of our knowledge no papers actually evaluate how Telometric measures

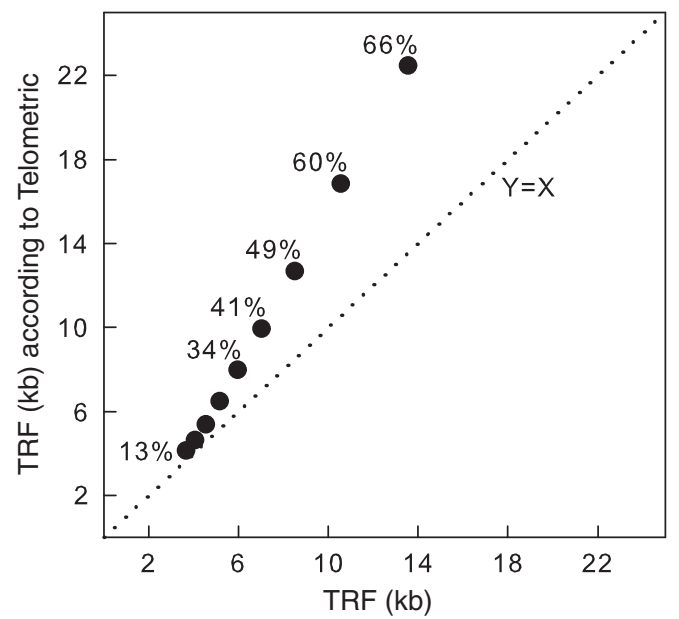

Figure 1 Telomere restriction fragment (TRF) length estimated using Telometric plotted against estimates obtained using an unbiased method. All data points were calculated from the same TRF electrophoresis distribution that was moved stepwise along the ladder towards higher molecular weights. Percentages near markers indicate by how much Telometric overestimates the TRF length. Note that the exact degree of overestimation will depend on the TRF distribution and hence these results cannot be used to correct data obtained using Telometric. See Supplementary Information for details, including the gel photograph and data file with calculations. telomeres in comparison with traditional analysis methods. Here, we further explain the cause of the bias in the telomere length estimates calculated by Telometric that we previously referred to. In the Supplementary Materials we provide a detailed illustration of telomere length measurement using Telometric compared with an unbiased technique based on the image analysis program Image $J$ that allows readers to repeat and verify our findings. In short, Telometric systematically deletes short telomeres and creates long telomeres from data read from the original gel image in order to create modified uniformly spaced data on which it calculates telomere length. Consequently, estimates of telomere length obtained using Telometric overestimate the telomere length, and this bias increases in absolute and relative terms with increasing telomere length. In our realistic example (see Supplementary Materials), this bias reached over $60 \%$ even at moderate telomere lengths (Figure 1). This suggests that comparisons of data between studies that use Telometric as opposed to other methods are problematic at best.

In conclusion, the telomere estimates obtained using Telometric are substantially biased and in its current form its usage should be avoided. However, we note that Telometric also has many useful features, and because it is an open-source program it is in fact possible for a Java programmer to modify it to produce unbiased estimates. As we continue to move forward in this exciting field, communication among labs and constant re-evaluation of ideas and techniques will be necessary; we hope that this letter contributes to this development.

\section{Conflict of interest}

The authors declare no conflict of interest.

MF Haussmann ${ }^{1}$, HM Salomons ${ }^{2}$ and S Verhulst ${ }^{2}$ ${ }^{1}$ Department of Biology, Bucknell University, Lewisburg, PA, USA and ' ${ }^{2}$ University of Groningen, Groningen, The Netherlands E-mail: mfh008@bucknell.edu

\section{References}

Grant JD, Broccoli D, Muquit M, Manion FJ, Tisdall J, Ochs MF (2001). Telometric: A tool providing simplified, reproducible measurements of telomeric DNA from constant field agarose gels. BioTechniques 31: 1314-1316.

Haussmann MF, Mauck RA (2008). New strategies for telomerebased age estimation. Mol Ecol Resourc 8: 264-274.

Horn T, Robertson BC, Gemmell NJ (2010). The use of telomere length in ecology and evolutionary biology. Heredity 105: 497-506.

Monaghan P (2010). Telomeres and life histories: the long and the short of it. Ann N Y Acad Sci 1206: 130-142.

Salomons HM, Mulder GA, van de Zande L, Haussmann MF, Linskens MHK, Verhulst S (2009). Telomere shortening and survival in free-living corvids. Proc R Soc B Biol Sci 276: 3157-3165. 